\title{
Prognostic significance of plasma D-dimer levels in patients with lung cancer
}

\author{
Osamu Taguchi, Esteban C Gabazza, Hiroki Yasui, Tetsu Kobayashi, \\ Masamichi Yoshida, Hiroyasu Kobayashi
}

\begin{abstract}
Background - The peripheral blood concentrations of several proteases of the clotting system have been shown to predict survival in patients with malignancy. A study was undertaken to investigate the independent value of the plasma levels of the $D$-dimer degradation product of fibrin before treatment for predicting prognosis in patients with lung cancer.

Methods - The study comprised 70 patients with lung cancer (49 non-small cell lung cancer and 21 small cell lung cancer). Plasma levels of D-dimer were measured using an enzyme immunoassay kit. Multivariate statistical analysis was carried out using the Cox's proportional hazards model.

Results - The median value of the plasma level of D-dimer differentiated two groups of patients with different outcomes: a group with a D-dimer level of $<150 \mathrm{ng} / \mathrm{ml}$ (low DD group) and those with D-dimer levels of $\geq 150 \mathrm{ng} / \mathrm{ml}$ (high DD group). Survival time was significantly better in patients in the low DD group than in those in the high DD group in all patients (hazard ratio for high $D D$ group $=4.7 ; 95 \%$ confidence interval (CI) 1.8 to 11.7$)$. The plasma levels of $\mathrm{D}$-dimer predicted survival independently from the clinical stage of disease, histological type, performance status, and tumour size (hazard ratio= $3.9 ; 95 \%$ CI 1.6 to 9.2 ).

Conclusions - These results suggest that plasma levels of D-dimer might be useful for predicting the clinical outcome in patients with lung cancer. However, further prospective studies are needed in a larger population to confirm these findings.

(Thorax 1997;52:563-565)
\end{abstract}

Keywords: D-dimer, lung cancer, prognosis.

Systemic activation of the clotting system occurs frequently in patients with lung cancer. ${ }^{1}$ The biological significance of the haemostatic abnormalities in cancer is not clear. There is some evidence to suggest that the capacity of neoplastic cells to activate the coagulation system and to express increased fibrinolytic activity facilitates their growth and contributes to their invasive and metastatic behaviour. ${ }^{2}$ Plasmin generation induced by tumour cells may influence their invasiveness and capacity to produce metastasis by its ability to degrade protein components of the extracellular matrix, activate latent enzymes such as type IV collagenase, and dissolve tumour associated fibrin clots. $^{2}$ Circulating markers of the fibrinolysis system are raised in patients with lung cancer and they have been shown to correlate with tumour burden, clinical progression, and the response to chemotherapy. ${ }^{3}$ The aim of this study was to investigate the independent value of the pretreated plasma levels of D-dimer fibrin degradation products for predicting prognosis in patients with lung cancer.

\section{Methods}

Seventy consecutive patients with lung cancer, of median age 65 years (range 20-83), admitted to the Mie University Hospital from July 1990 to December 1991 took part in the study. There were 49 cases with non-small cell lung cancer (non-SCLC) and 21 with small cell lung cancer (SCLC). Clinical staging was performed according to the new international staging system. Patients underwent curative surgery $(n=20)$ or combination chemotherapy followed by radiotherapy $(n=50)$. Venous blood samples were taken 1-5 days (median two days) before starting any treatment and stored at $-80^{\circ} \mathrm{C}$ until needed. Plasma levels of D-dimer were determined using an enzyme immunoassay kit (Dimertest, Agen, Mountain View, California, USA). D-dimer levels were also measured in blood samples from age matched healthy volunteers $(n=40)$ and from patients with benign pulmonary disease $(n=25)$. There was a history of smoking in 50 patients with lung cancer, in 15 with benign disease, and in 10 healthy subjects. The intra-assay and inter-assay precision of D-dimer concentrations was $5.4 \%$ and $9.5 \%$, respectively. Written consent was obtained from all subjects and the investigation was carried out according to the conditions of the Helsinki declaration.

STATISTICAL ANALYSIS

Data are expressed as mean (SE) values. The Student's $t$ test was used to compare means between groups where appropriate. A p value of $<0.05$ was considered to be statistically significant. Survival time was calculated from the date of diagnosis of the disease to the date of death from the disease. Patients lost to follow up and deaths from different causes were considered as censored observations. The independent prognostic value of $\mathrm{D}$-dimer and that of other factors were analysed by Cox's proportional hazards model. ${ }^{4}$ The cut off value 
of D-dimer levels for distinguishing prognostic groups was taken as the median value calculated by the box-and-whisker plot.

\section{Results}

Patients were followed up for a total of six years (median 15 months; range 2-66 months); 27 were censored observations. The plasma level of D-dimer was significantly higher $(\mathrm{p}<0.0001)$ in patients with lung cancer $(276.7$ (34.2) $\mathrm{ng} / \mathrm{ml}$ ) than in healthy controls (52.8 (3.9) $\mathrm{ng} / \mathrm{ml}$ ) or in those with benign disease (81.1 (7.5) $\mathrm{ng} / \mathrm{ml})$. A significant correlation was found between plasma levels of $\mathrm{D}$-dimer and survival time $(r=-0.6 ; \mathrm{p}<0.0001)$. The median plasma level of $\mathrm{D}$-dimer differentiated between two groups of patients with different outcomes: a group with a D-dimer level of $<150 \mathrm{ng} / \mathrm{ml}$ (low DD group) and another with a D-dimer level of $\geq 150 \mathrm{ng} / \mathrm{ml}$ (high DD group). Survival time was significantly longer in all cancer patients in the low DD group than in those in the high DD group (hazard ratio $(\mathrm{HR})=4.7 ; 95 \%$ confidence interval $(\mathrm{CI}) 1.8$ to $11.7 ; \mathrm{p}<0.0005$; fig $1 \mathrm{~A}$ ). There was a significant difference in survival between patients with low and high plasma levels of D-dimer in those with non-SCLC (HR for high DD group $=4.6$; $95 \%$ CI 1.8 to 11.6 ; $p<0.002$; fig 1B) but not in those with SCLC (data not shown). Prognosis was also better in the low DD group than in the high DD group in patients with non-SCLC with extensive disease $(\mathrm{HR}=6.4 ; 95 \%$ CI 1.4 to 28.6 ; $\mathrm{p}<0.02$; fig 1C). The numbers of patients with non-SCLC with limited disease and of SCLC cases were not sufficient to allow statistical evaluation. The plasma level of $\mathrm{D}$-dimer as a binary variable (high or low) predicted survival in all patients independently from other prognostic factors $(\mathrm{HR}=3.9 ; 95 \%$ CI 1.6 to $9.2 ; \mathrm{p}=0.001)$ including clinical stage, performance status, and tumour size.

\section{Discussion}

Activation of fibrinolysis secondary to activation of coagulation is a well recognised complication in patients with lung cancer. ${ }^{5}$ The biological significance of the activation of the clotting system and fibrinolysis in cancer has recently been discussed with regard to the potential role of these pathways in the pathogenesis of malignancy for their ability to regulate tumour growth and dissemination. ${ }^{6}$ In this regard, anticoagulant therapy was reported to suppress the invasion of cancer cells in experimental models and to show survival benefit in some types of lung tumour in humans when used in combination with other cytotoxic drugs. ${ }^{7}$ Fibrin deposits in lung cancer tissues may promote cell proliferation and neovascularisation of the growing tumour, and they may protect tumour cells from immune or chemotherapeutic attacks and favour their capillary implantation. ${ }^{8}$ On the other hand, the fibrin framework in primary tumours may also block the entrance of tumour cells into the circulation. ${ }^{8}$ However, lung cancer cells also
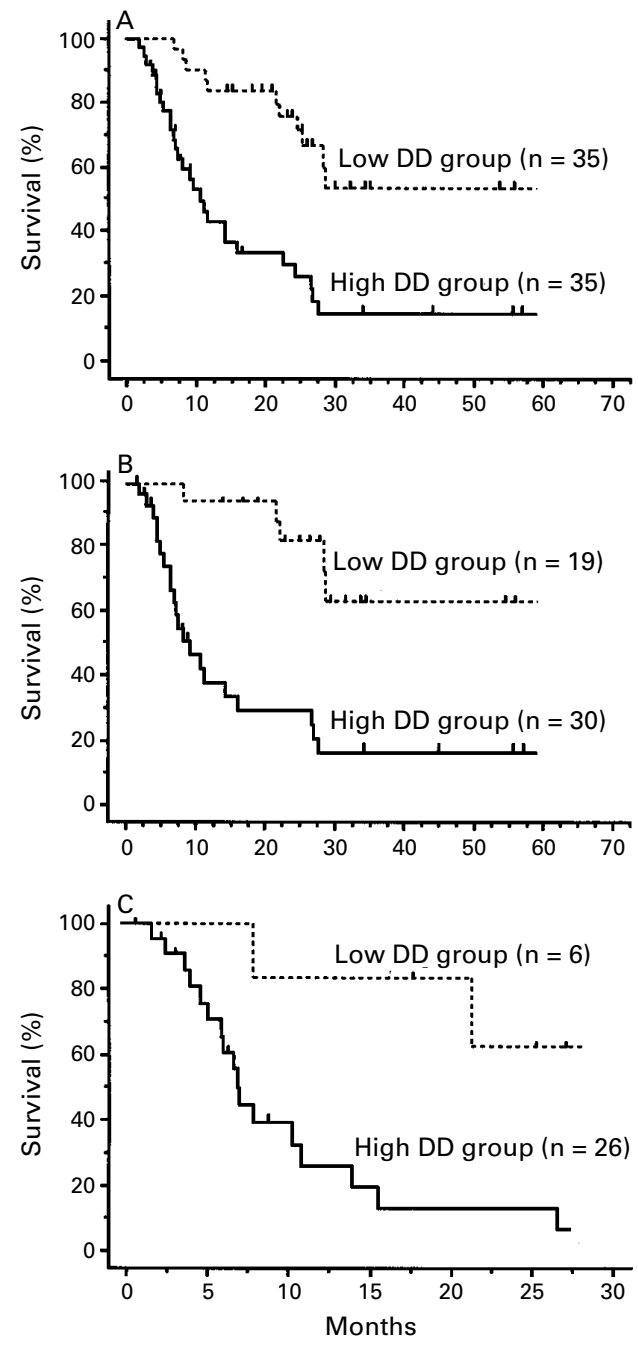

Figure 1 Effect of plasma levels of $D$-dimer (DD) on survival in (A) all patients with lung cancer, (B) all patients with non-small cell lung cancer (non-SCLC), and $(C)$ all patients with non-SCLC with extensive disease. Vertical lines represent censored observations.

express urokinase and tissue plasminogen activators which, by acting on plasminogen, generate plasmin - the active serine protease of the fibrinolysis system - that may act on fibrin in the primary tumours increasing the detachment of cells and their penetration into the circulatory channels. ${ }^{9}$ In addition, plasmin may promote tumour invasion by degrading various protein components of the extracellular matrix, either directly or through the activation of various procollagenases such as procollagenase type IV secreted by tumour cells. ${ }^{9}$ In this study the blood concentration of the fibrin degradation fragment $\mathrm{D}$-dimer was found to be an independent predictor of survival in patients with lung cancer. The prognostic significance of this fibrinolytic marker may reflect the participation of plasmin in the process of tumour spread. In agreement with this, the plasma levels of protease components of the fibrinolysis system have also been found to be abnormally raised and to predict survival in lung cancer patients. ${ }^{10}$ However, further prospective studies 
must be carried out in a larger population to confirm our preliminary findings.

1 Bick RL. Coagulation abnormalities in malignancy: a review.

Semin Thromb Hemost 1992;18:353-72.
2 Kwaan HC, Keer HN. Fibrinolysis and cancer. Semin Thromb Hemost 1990;16:230-5.

3 Gabazza EC, Taguchi O, Yamakami T, Machishi M, Ibata $\mathrm{H}$, Suzuki S. Evaluating the prethrombotic state in lung cancer using molecular markers. Chest 1993;103:196-200. 4 Cox DR. Regression models and life tables. 7 R Stat Soc 1972;187:187-220

5 Rickles FR, Edwards RL. Activation of blood coagulation in cancer: Trousseau's syndrome revisited. Blood 1984; 62:14-31.
6 Constantini V, Zacharski LR. Fibrin and cancer. Thromb Haemost 1993; 69:406-14

7 Zacharski LR, Memoli VA, Constantini V, Wojtukiewicz MZ, Ornestein DL. Clotting factors in tumor tissue: implications for cancer therapy. Blood Coagul Fibrinolysis 1990;1:71-8.

8 Gasic GJ, Tuszynski GP, Gorelik E. Interaction of the hemostatic and immune systems in the metastatic spread of tumor cells. Int Rev Exp Pathol 1986;29:173-212.

9 Tryggvason KH, Salo T. Proteolytic degradation of extracellular matrix in tumor invasion. Biochim Biophys Acta cellular matrix in tum

10 Wojtukiewicz MZ, Zacharski LR, Moritz TE, Edwards RL, Rickles FR. Prognostic significance of blood coagulation tests in carcinoma of lung and colon. Blood Coagul Fib rinolysis 1992;3:429-37.

\title{
Attenuation of oxidant/antioxidant imbalance during treatment of exacerbations of chronic obstructive pulmonary disease
}

\author{
I Rahman, E Skwarska, W MacNee
}

\begin{abstract}
Background - An oxidant/antioxidant imbalance is thought to occur in patients with chronic obstructive pulmonary disease (COPD). It has recently been shown that during exacerbations of COPD the antioxidant capacity and protein sulfhydryls of plasma are lower and the levels of products of lipid peroxidation are higher than in age matched healthy subjects. The aims of this study were to confirm these data and to measure the time course of these changes.

Methods - The plasma Trolox equivalent antioxidant capacity (TEAC), protein sulfhydryls, and products of lipid peroxidation were measured throughout the course of treatment in 13 patients who presented with an acute exacerbation of COPD.
\end{abstract}

Results - TEAC values (mmol/1) were low on admission (mean $0.67,95 \%$ confidence interval (CI) 0.32 to 0.88 ; $p<0.05$ ) and had increased by discharge $(0.98,95 \%$ CI 0.88 to $1.2 ; \mathrm{p}<0.05)$ but still remained lower than in healthy subjects $(1.33,95 \%$ CI 1.11 to 1.65$)$. There was also restoration of plasma protein sulfhydryl levels (mmol/1) from admission $(0.32,95 \%$ CI 0.20 to 0.43$)$ to discharge $(0.49,95 \% \mathrm{CI} 0.42$ to 0.62 , p $<0.001)$ to levels similar to those in healthy subjects $(0.52,95 \%$ CI 0.43 to 0.65$)$. Products of lipid peroxidation, measured as thiobarbituric acid-malondialdehyde adducts $(\mu \mathrm{mol} / 1)$, were significantly higher $(2.08,95 \%$ CI 1.8 to 2.5$)$ than in control subjects $(1.3,95 \% \mathrm{CI} 0.85$ to $1.32 ; \mathrm{p}<0.01)$ and returned to normal values by the time of discharge $(1.2,95 \%$ CI 0.88 to 1.29$)$.

Conclusions - These data confirm the presence of a profound oxidant/anti- oxidant imbalance in the blood of patients with acute exacerbations of COPD which returns towards normal values during the course of treatment.

(Thorax 1997;52:565-568)

Keywords: oxidants, antioxidants, chronic obstructive pulmonary disease, lipid peroxides.

An oxidant/antioxidant imbalance is thought to play a part in the pathogenesis of chronic obstructive pulmonary disease (COPD). ${ }^{1} \mathrm{Ci}$ garette smoke and the release of reactive oxygen intermediates (ROI) from circulating neutrophils and airspace macrophages are major sources of oxidant stress in patients with COPD. ${ }^{1}$

There is, however, a paucity of data on the oxidant/antioxidant imbalance in patients with COPD, particularly during exacerbations. We have recently shown that the antioxidant capacity of plasma is lower during exacerbations of COPD than in age matched healthy subjects or patients with clinically stable COPD, suggesting increased oxidant stress. ${ }^{2}$ This previous study was conducted in parallel groups of patients with either clinically stable or exacerbations of COPD. In the present study our aim was to assess the time course of the changes in markers of oxidant stress in plasma in individual patients during the course of an exacerbation of COPD.

\section{Methods}

STUDY POPULATION

Thirteen patients (six men) of mean (SD) age 69 (8) years who presented with an acute exacerbation of COPD were studied. The diagnosis of COPD was made by a respiratory 


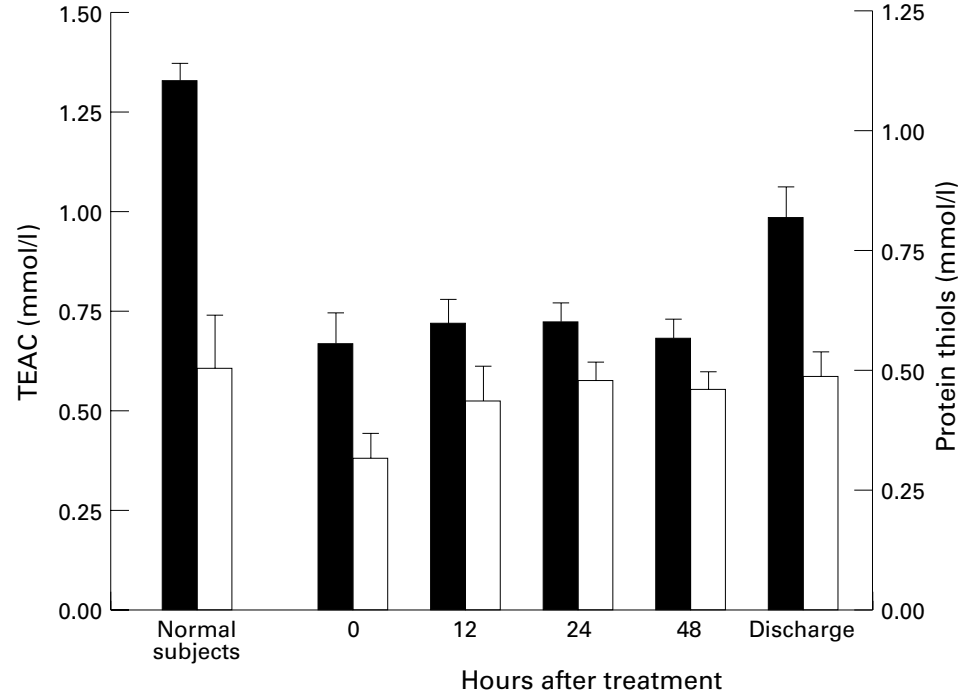

Figure 1 Time course of the changes in plasma Trolox equivalent antioxidant capacity (TEAC); solid columns) and protein thiols (open columns) in normal subjects and patients with exacerbations of COPD. physician on the basis of current or ex-smoking and severe, largely irreversible, airways obstruction $(<15 \%$ improvement in baseline $\mathrm{FEV}_{1}$ in response to inhaled $\beta_{2}$ agonist). An exacerbation of COPD was defined as an increase above the patient's normal symptoms in two of the following: (1) breathlessness; (2) cough or sputum production; (3) sputum purulence, which was severe enough to warrant admission.

Six of the 13 patients had been started on oral corticosteroid therapy (20-40 mg prednisolone) during the week before admission. Prior to admission the other medications in these patients consisted of inhaled steroids and bronchodilator therapy in the form of $\beta_{2}$ agonists and/or ipratropium bromide. Treatment during the admission consisted of oxygen (1-3 $1 /$ min by nasal prongs), nebulised $\beta_{2}$ agonists, and ipratropium and continuation of corticosteroids in those who had received this treatment prior to admission. Six of the 13 patients were smokers and seven were ex-smokers who had quit smoking between six weeks and four years before their admission.

Eighteen healthy non-smokers (eight men) of mean (SD) age 59 (7) years randomly selected from the hospital staff who had no history of lung diseases were used as control subjects.

Blood samples were withdrawn from the patients within three hours of admission with an exacerbation of COPD and at 12, 24, and 48 hours thereafter. A further sample was taken 5-10 days later when the patient's condition was considered clinically stable enough for discharge. None of the patients smoked during their hospital admission. In the normal subjects blood samples were obtained on two occasions one week apart.

The study was approved by the local ethical committee and all patients gave written informed consent.
METHODOLOGY

For all of the assays described below $10 \mathrm{ml}$ of venous blood was withdrawn into a lithiumheparin tube, centrifuged $(250 \mathrm{~g})$, and the plasma removed and used immediately for the following assays.

Trolox equivalent antioxidant capacity (TEAC) The plasma antioxidant capacity was measured by the method of Miller et al. ${ }^{3}$ The Trolox equivalent antioxidant capacity (TEAC) was calculated by defining the concentration of Trolox in $\mathrm{mmol} / \mathrm{l}$ having the equivalent antioxidant capacity to a $1.0 \mathrm{mmol} / 1$ sample of the plasma under investigation.

\section{Products of lipid peroxidation}

The concentration of products of lipid peroxidation in plasma as thiobarbituric acid (TBA)-malondialdehyde (MDA) adducts was measured by the method described by Yagi. ${ }^{4}$ The final result was expressed as mol TBAMDA adducts formed/litre of plasma.

Protein sulfhydryl and protein carbonyl assays Protein thiols were measured using the method of Ellman ${ }^{5}$ and protein carbonyls were assayed by the method described by Rodney and coworkers. ${ }^{6}$

We have already confirmed the sensitivity and reproducibility of these assays. ${ }^{2}$

\section{STATISTICAL ANALYSIS}

The data are expressed as mean and 95\% confidence intervals (CI). Differences between mean values were assessed by a one way analysis of variance using Tukey's method.

\section{Results}

Arterial blood gas tensions on admission breathing oxygen (1-3 1/min by nasal prongs) were $\mathrm{PaO}_{2} 10.6(0.9) \mathrm{kPa}, \mathrm{PaCO}_{2} 6.5(0.5) \mathrm{kPa}$, $\mathrm{H}^{+}$ion 42.7 (1.6) nmol/l. None of the patients had clinical or radiological evidence of a pneumonia. Serum albumin and haemoglobin concentrations were $39.5(1.3) \mathrm{g} / \mathrm{l}$ and $130.6(15.3)$ $\mathrm{g} / \mathrm{l}$, respectively. The mean (SD) $\mathrm{FEV}_{1}$ (\% of predicted values) was 23 (7)\% on admission, $26(8) \% 24$ hours after admission, 31 (10)\% at 48 hours, and 31 (6)\% at discharge 5-10 days after admission.

The TEAC of plasma was lower in the patients at the time of their presentation with an acute exacerbation of COPD (0.67, 95\% CI 0.32 to $0.88 \mathrm{mmol} / \mathrm{l}$ ) than in normal age and sex matched healthy control subjects (1.33, $95 \%$ CI 1.11 to $1.65 \mathrm{mmol} / \mathrm{l}, \mathrm{p}<0.05$; fig 1 ). TEAC values were lower in those patients who were current smokers (mean $0.53 \mathrm{mmol} / 1,95 \%$ CI 0.31 to $0.73 ; \mathrm{n}=7$ ) than in those who were ex-smokers $(0.81 \mathrm{mmol} / 1,95 \%$ CI 0.68 to 0.90 , $\mathrm{n}=6 ; \mathrm{p}<0.05)$. Patients who received treatment with steroids prior to admission had similar TEAC values $(0.65 \mathrm{mmol} / 1,95 \%$ CI 0.41 to $0.88 ; n=6$ ) to those who had not received 


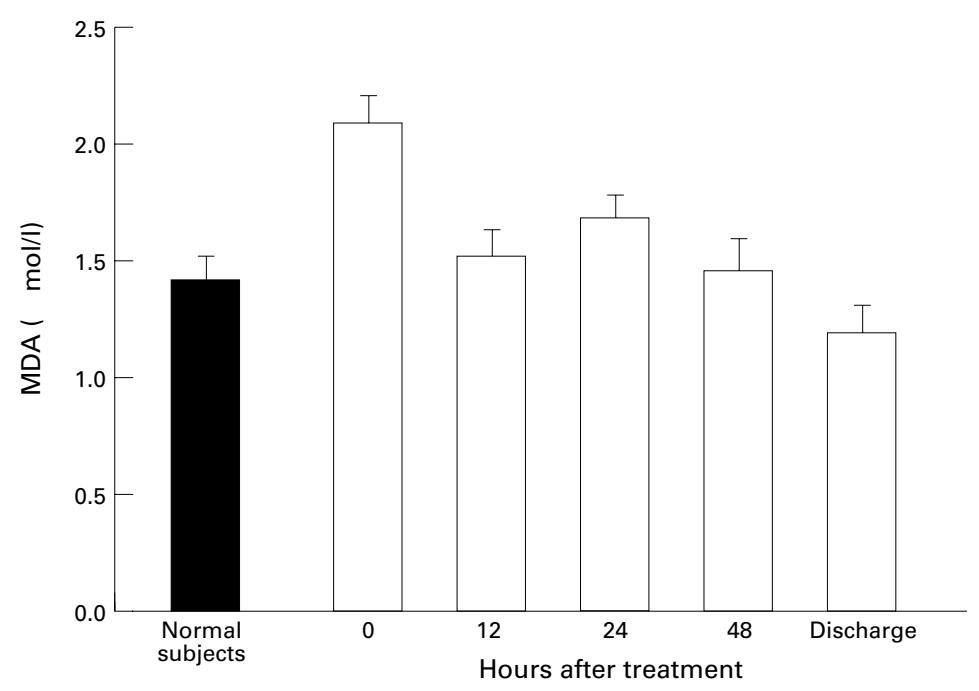

Figure 2 Time course of the changes in products of lipid peroxides, measured as TBA$M D A$ adducts, in patients with acute exacerbations of COPD.

\section{Discussion}

In a previous parallel group study we reported that the antioxidant capacity of plasma was reduced in smokers and in patients with exacerbations of COPD compared with age matched healthy subjects. ${ }^{2}$ This was associated with increased superoxide anion generation by circulating neutrophils, which diminished by the time of discharge. In this study we confirm our earlier observations and provide further insights into the time course of the change in oxidant/antioxidant imbalance in acute exacerbations of COPD.

A persistently decreased antioxidant capacity was seen in the plasma of patients with an acute exacerbation of COPD for at least 48 hours following their admission, with a rise at the end of the exacerbation when they were considered to be clinically stable enough for discharge. However, in contrast to patients with clinically stable COPD who were studied at least six weeks after their last exacerbation, ${ }^{2}$ TEAC values had not returned to normal levels.

A low TEAC in plasma suggests increased oxidant stress in the blood. As a result of oxidant stress, lipid peroxides are formed due to the peroxidation of unsaturated fatty acids present on cell membranes. ${ }^{7}$ Our observation of increased levels of products of lipid peroxidation in plasma, together with a fall in antioxidant capacity in this and our previous study, strongly supports our contention that there is increased oxidative stress in patients with acute exacerbations of COPD. The increase in products of lipid peroxidation in plasma fell rapidly to normal values within 12 hours of admission and remained at these levels throughout the study. In contrast, plasma TEAC had not completely recovered to normal levels by the time of discharge.

The decrease in the TEAC was more pronounced in patients with a current smoking history than ex-smokers, which is in agreement with our earlier observations of a dramatic decrease in TEAC during smoking. ${ }^{2}$ We also confirmed the results of our previous study that pretreatment with steroids did not affect TEAC values in patients with acute exacerbations of COPD.

In our previous study we found a correlation between plasma TEAC, the release of reactive oxygen intermediates (ROS) from circulating neutrophils, and protein sulfhydryls, suggesting a mechanism for the fall in plasma TEAC levels. ${ }^{2}$ Several components of plasma contribute to plasma TEAC. Albumin, which has a sacrificial sulfhydryl group, is thought to account for two thirds of the plasma TEAC. ${ }^{3}$ Many proteins such as ceruloplasmin, transferrin, and small antioxidant molecules such as non-protein thiols, vitamins $\mathrm{C}$ and $\mathrm{E}$, and uric acid account for the remainder of the plasma TEAC. It has been reported that smoking is associated with a depletion of vitamin $\mathrm{C}$ and E levels in the blood. ${ }^{8}$ The lower plasma TEAC in patients with acute exacerbations of COPD with a smoking history may therefore result from depletion of such antioxidant molecules. A dramatic fall in protein thiols is confirmed in this study in patients with acute exacerbation 
of COPD. However, this depletion of protein thiols was restored during treatment of the exacerbation, beginning at 12 hours, and was back to normal levels by the time of discharge, while TEAC levels recovered significantly, but not to normal values, by the time of discharge. The increased oxidative stress measured as increased products of lipid peroxidation and decreased protein thiols normalised during the first 12 hours of treatment. However, a persistent depletion of other antioxidant molecules during exacerbations of COPD may account for the incomplete recovery of TEAC at or after 48 hours of treatment. Further studies are required to investigate this hypothesis.

The mechanism of the restoration of the plasma protein thiols during treatment of the exacerbation of COPD is unknown. Prednisolone stimulates the synthesis of thiols such as glutathione in the liver, ${ }^{9}$ thus providing thiol groups to replenish protein thiols. However, in this relatively small group of patients there did not appear to be any relationship between the changes in plasma TEAC or protein thiols and any specific treatment, including corticosteroids. However, the possible role of steroids or other medications in inducing the recovery of protein thiols, and the antioxidant capacity of plasma during treatment of the exacerbation of COPD, requires further study in a larger group of patients. In addition, studies to monitor the levels of antioxidant molecules such as vitamins $\mathrm{C}$ and $\mathrm{E}$, uric acid and beta-carotene during the course of acute exacerbations of COPD will help to elucidate the relationship between the fall in the antioxidant capacity of the plasma and the pathogenesis of exacerbations of COPD. Such studies may lead to the development of novel therapeutic interventions with antioxidant therapy in this condition.

These data add further support to the concept of an increase in oxidant stress in exacerbations of COPD, and point the way to further studies in this area.

This study was supported by the Chest, Heart and Stroke Association (Scotland) and the Norman Salvesen Emphysem Research Trust. The authors wish to thank Eileen Neal for typing the manuscript.

1 Rahman I, MacNee W. Oxidant/antioxidant imbalance in smokers and chronic obstructive pulmonary disease. Thorax smokers and chro

2 Rahman I, Morrison D, Donaldson K, MacNee W. Systemic oxidative stress in asthma, COPD and smokers. Am $\mathcal{F}$ Respir oxidative stress in asthma, COPD
Crit Care Med 1996;154:1055-66.

3 Miller NJ, Rice-Evans C, Davies MJ, Gopinathan V, Milner A. A novel method for measuring antioxidant capacity and its application to monitoring the antioxidant status in premature babies. Clin Sci 1993;84:407-12

4 Yagi K. A simple fluorometric assay for lipoperoxide in blood plasma. Biochem Med 1976;15:212-6.

5 Ellman GL. Tissue sulfhydryl groups. Arch Biochem Biophys 1959;82:70-7

6 Rodney LL, Galland D, Oliver CN, Amici A, Climent I, Lenz A, et al. Determination of carbonyl content in oxidatively modified proteins. Methods Enzymol 1990;186:464-78.

7 Pryor WA, Godber SS. Non-invasive measures of oxidative stress status in humans. Free Radic Biol Med 1991;10: 177-84.

8 Mazzetti A, Lapenna D, Pierdomenico SD, Calafiore AM Costantini F, Riario-Sforza G, et al. Vitamin E, C and lipid peroxidation in plasma and arterial tissue of smokers and non-smokers. Atherosclerosis 1995;112:91-9.

9 Speck RF, Schrauz C, Lauterbrugh BH. Prednisolone stimulates hepatic glutathione synthesis in mice. F Hepatol 1993 18:62-7. 